\title{
Impact of particle sizes on the microwave absorption properties of nano-sized Carbon black/epoxy composites
}

\author{
Sandeep Kumar Singh ${ }^{1 *}$, Amit Kumar Yadav ${ }^{1}$, Ranu Pal ${ }^{1}$, M. J. Akhtar ${ }^{1,2}$ and Kamal K. Kar ${ }^{1,3}$ \\ ${ }^{I}$ Department of Materials Science Programme, Indian Institute of Technology Kanpur, Kanpur, India \\ ${ }^{2}$ Department of Electrical Engineering, Indian Institute of Technology Kanpur, Kanpur, India \\ ${ }^{3}$ Advanced Nanoengineering Materials Laboratory, Department of Mechanical Engineering and Materials Science \\ Programme, Indian Institute of Technology Kanpur, Kanpur 208016, India
}

*Corresponding author

DOI: $10.5185 / \mathrm{amp} .2018 / 7008$

www.vbripress.com/amp

\begin{abstract}
The nano-sized carbon black (NCB) with particle sizes of 15, 25, 55 and $65 \mathrm{~nm}$ are added in epoxy matrix in order to investigate the impact of particle sizes on the microwave absorption properties of the nano composites. The NCB powders were characterized by X-ray diffraction and Scanning electron microscopy (SEM) for identification of impurity phases. The complex permittivity spectra were recorded for the composites in the frequency range of 8.2-12.4 GHz. The results revealed that the particle sizes had substantial impact on the electromagnetic properties. On decreasing the particle size, the complex permittivity increases first up to certain extent then decreases. Interestingly, the particle size dependent tangent loss of NCB/epoxy composites shows that $25 \mathrm{~nm}$ size is most suited for high loss characteristics, whereas, losses decreases with size $<25 \mathrm{~nm}$, which is attributed to high surface area of the particles. In addition, NCB/epoxy composite with $25 \mathrm{~nm}$ particle size illustrated substantial EM absorption efficiency at a frequency of $9.5 \mathrm{GHz}$, with RL reaching $-13.2 \mathrm{~dB}$ and reflection loss below $-10 \mathrm{~dB}$ up to $2.5 \mathrm{GHz}$ with thickness of $2.0 \mathrm{~mm}$. The effect of particle sizes on the electromagnetic properties was also discussed. Copyright (C) 2018 VBRI Press.
\end{abstract}

Keywords: Nano-sized carbon black, particle size, composite, electromagnetic property, microwave absorption.

\section{Introduction}

Demand for microwave absorbers has increased dramatically owing to its potential application in military, telecommunication, satellite communication and electronic devices that causes problem like electromagnetic interference [1]. Therefore, numerous materials have attracted for the development of absorber. Since, Nano-sized Carbon black (NCB) possess high conductivity and high losses, therefore widely used as EM absorbing material [2]. The design of absorber depends on electromagnetic (EM) properties of material such, as complex permittivity [3]. In the commercial utilization of absorber, the permittivity is a crucial parameter that improves the microwave absorption ability in terms of broad bandwidth, thin and light weight absorber [4]. It is obvious that features like particle size and the composition of the particles, are key parameters for determining the permittivity [5]. However, the report about the impact of particle sizes on the EM properties of NCB based absorber in microwave frequency has not been explored.

The objective of the present study is to explore how particle size of $\mathrm{NCB}$ influences the electromagnetic
(EM) properties. In the present paper, we report the EM properties of NCB/epoxy composites with different NCB particle sizes ranging from $65-15 \mathrm{~nm}$ in the $\mathrm{X}$ band (8.2-12.4 GHz) frequency, in addition the impacts of particle sizes are well explained in detail.

\section{Experimental}

The commercially available conductive nano seized carbon black (NCB) of four dissimilar grades N220, N330, N660, and N774 by Kankani Brothers Ltd. India were chosen for NCB/epoxy nano-composite (NC) fabrication. Epoxy resin (PG100, obtained from M/S Resinova Chemie, India), binder PHY161 (received from M/S Resinova Chemie, India) in weight ratio 10:1 and 20 wt. \% of NCB were used for composites preparation. Dispersion is a crucial key parameter to fully explore the properties of composites. Therefore, NCB was initially dispersed in ethanol via magnetic stirrer for an hour. Then the solution was mixed with epoxy resin and further stirred for half hour. Finally, binder was added in the mixture and poured in mould of dimension $(22.86 \mathrm{~mm} \times 10.16 \mathrm{~mm} \times 2.5 \mathrm{~mm})$. The pressure of $0.25 \mathrm{MPa}$ at room temperature was applied 
to obtain bubble free composite. At last, sample placed in an oven at $65{ }^{\circ} \mathrm{C}$ for 4 hours in order to make flat rectangular sheet. Details of the fabricated composite samples are provided in Table 1.

Table 1. Physical property of NCB and details of prepared NCB/epoxy composite samples.

\begin{tabular}{cccc}
\hline $\begin{array}{c}\text { Composite } \\
\text { sample }\end{array}$ & $\begin{array}{c}\text { NCB ASTM } \\
\text { number }\end{array}$ & $\begin{array}{c}\text { NCB content } \\
\text { (wt. \%) }\end{array}$ & $\begin{array}{c}\text { Particle size } \\
(\mathrm{nm})\end{array}$ \\
\hline Epoxy & - & - & - \\
NC220 & N220 & 20 & 15 \\
NC330 & N330 & 20 & 25 \\
NC660 & N660 & 20 & 55 \\
NC774 & N774 & 20 & 65 \\
\hline
\end{tabular}

\section{Results and discussions}

\section{X-Ray Diffraction}

Fig. 1. represents the XRD spectra of N220, N330, N660 and N774 graded NCB powders [6]. It is confirmed that NCB powders are impurity free as no additional peaks present in XRD pattern. This is essential information in the study of particle size impact on EM properties; means there would be only NCB particle contribution on effective properties.

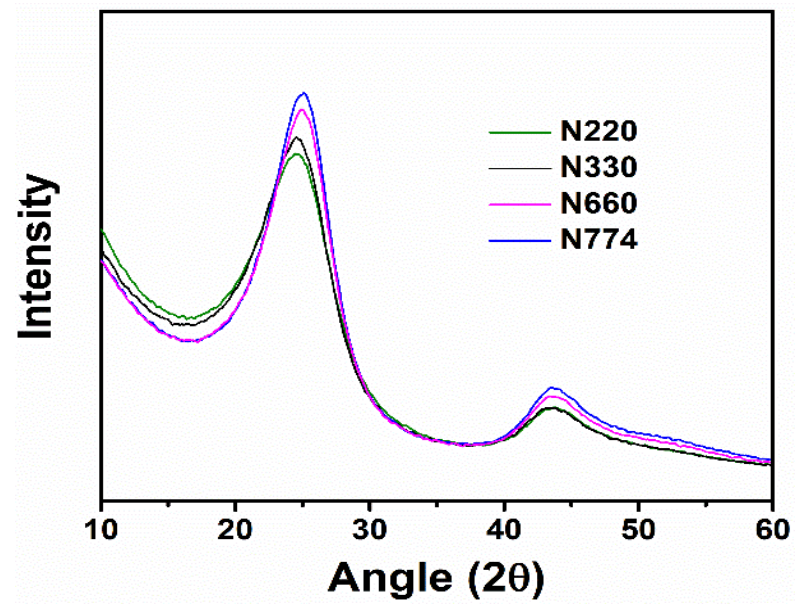

Fig. 1. X-ray Diffraction pattern of N220, N330, N660 and N774 graded NCB powders.

\section{Scanning electron microscopy}

Dispersion is a crucial parameter to explore the composite properties. Therefore, in order to study the dispersion of NCB particles in epoxy matrix, scanning electron microscopy (SEM; Zeiss EVO MA-15) measurement was performed. Fig 2 (a) - (e) shows the SEM images of epoxy, NC774, NC660, NC330 and NC220 samples, respectively. It is worth to mention that dispersion improves with decreasing particles size and good homogeneous dispersion was achieved with $25 \mathrm{~nm}$ of particle size (Fig 2(d)). NC220 with $15 \mathrm{~nm}$ of size, poor dispersion has been achieved and agglomeration of nano particles were noticed in the epoxy media as shown in Fig 2(e). This agglomeration is attributed to smaller nano-meter size particles that exhibits extremely high surface area. Therefore, it is expected that due to this agglomeration composite properties would behave abruptly.
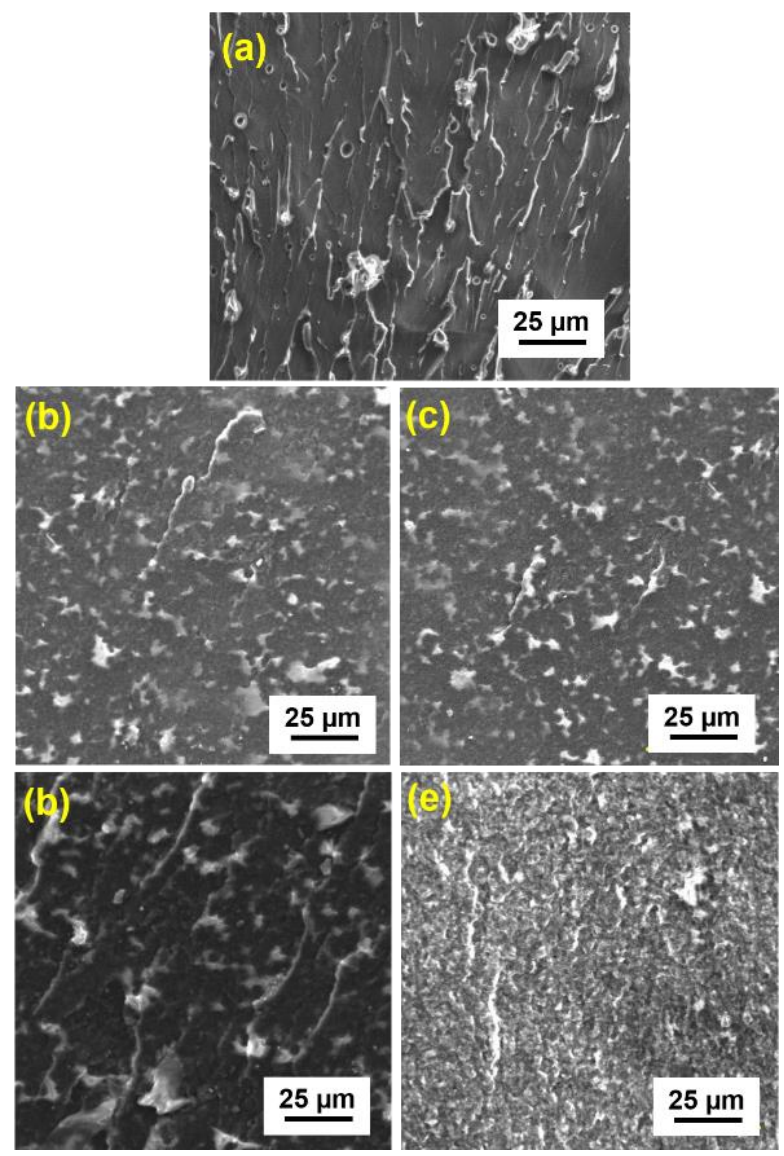

Fig. 2. SEM images of (a) epoxy, (b) NC774, (c) NC660, (d) NC330 and (e) NC220 composites.

\section{Complex permittivity}

The samples were scrutinized in the $\mathrm{X}$ band (8.2-12.4 $\mathrm{GHz}$ ) with the aim of observing the impact of the particle sizes on the complex effective permittivity. It is well proven that real part of permittivity is associated with amount of polarizations in the composite that originate from dipolar and interfacial polarizations whereas, imaginary permittivity is associated with dielectric losses. The data obtained from these samples demonstrate that particle exhibits substantial influence on the complex effective permittivity. The complex effective permittivity increases first and achieved the maximum change then decreases, with decrease in particles size in nanometer range. The real and imaginary permittivity of NC330 with particle size $25 \mathrm{~nm}$ turns out to be higher than the NC220, NC660 and NC774 as shown in Fig 3(a) - (e). However, NC220 with $15 \mathrm{~nm}$ particle size shows lowest permittivity (Fig 3(e)). Overall, $25 \mathrm{~nm}$ size is most suited for the significant enhancement in permittivity and losses. This can be understood on the fact that the particle sizes are different therefore; particle density would be higher for smaller sizes at a certain weight. Further, due to the small nano 
sizes of the particle they exhibits high surface area [7]. This high surface area enables more and more interaction between particle and incoming electromagnetic (EM) waves at the same time high particle density will make more conductive channel to facilitate large number of easy paths for EM waves to propagate inside the composites, which provide composites with improved complex effective permittivities [8-9]. However, it is anticipated that, the NCB with smaller particle size $(15 \mathrm{~nm})$ induces agglomeration that leads to non-uniform distribution of NCB in resin matrix due to extremely high surface area as shown in SEM images (Fig 3(e)). This concludes that the apparent filler distribution decreases with decreasing particle sizes below $25 \mathrm{~nm}$.
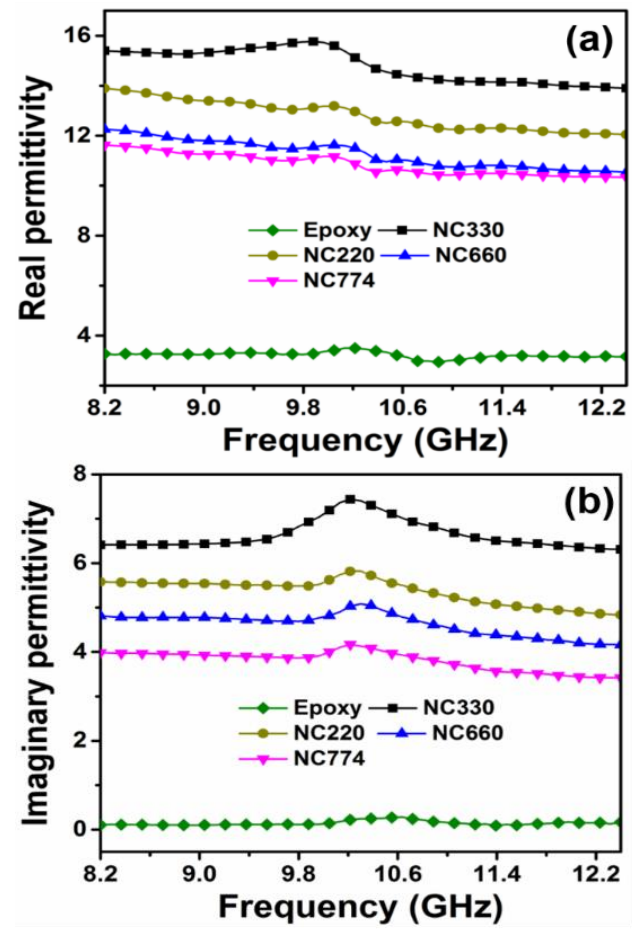

Fig. 3. Frequency dependent (a) reap permittivity and (b) imaginary permittivity of NC220, NC330, NC660 and NC774.

\section{Loss Tangent}

The dielectric loss tangent $(\tan \delta)$ that is typically expressed as the ratio of imaginary permittivity $\left(\varepsilon^{\prime \prime}\right)$ to real permittivity $\left(\varepsilon^{\prime}\right)$ is a measure of energy loss in a material [10]. It is an important parameter as it is associated with the attenuation factor which transforms the stored energy into dissipative heat. Therefore, high loss tangent indicate that material is very lossy, which is much needed parameter for designing an absorber. From Fig. 4 (a) it can be seen that the loss tangent first increases, then decreases, with decrease of particle size in nanometer range and attained maximum for NC330. Thus, results from loss tangent show that NC330 is lossier and most suited for various absorber applications.

A closer inspection of particle size effect on loss tangent has shown in Fig 4(b). The change in loss tangent calculated with respect to NC774. It is worth to mention that the change in loss tangent increases first and reached highest $39 \%$ for NC330 with size $25 \mathrm{~nm}$, then reduces, with decrease in particle sizes. In addition, due to lossier characteristics of N330, it is highly expected that it would certainly lower the percolation threshold. This suggest that $25 \mathrm{~nm}$ size of carbon black could be a potential candidate for designing the light weight and thin microwave absorber.
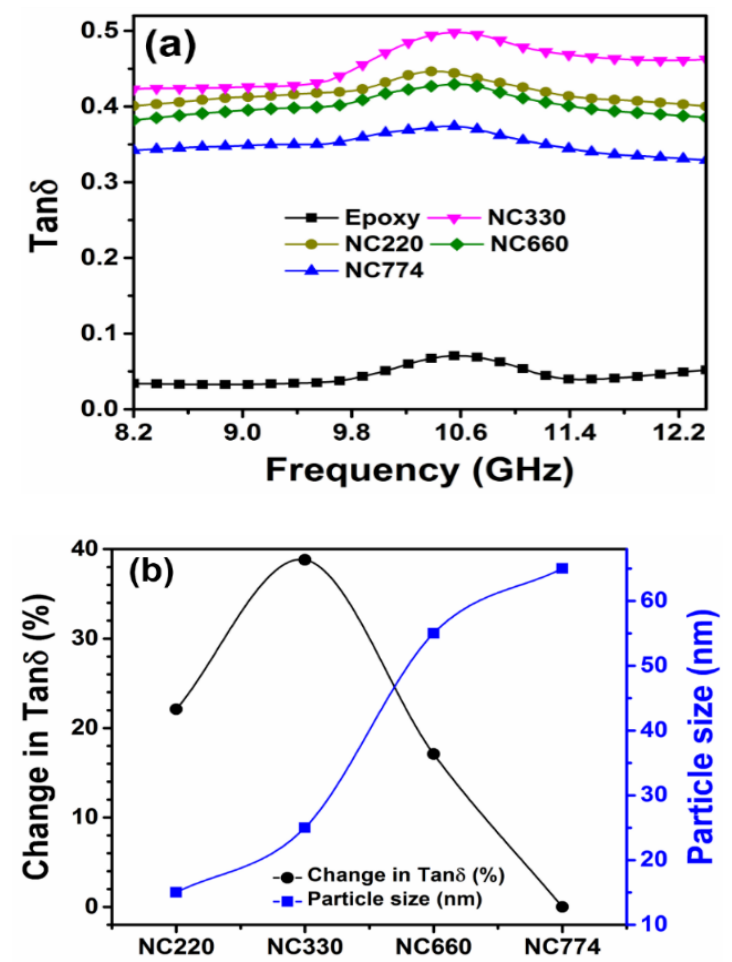

Fig. 4. (a) Frequency dependent loss tangent responses and (b) Variation of change in loss tangent with particle sizes of NC220, NC330, CN660 and CN774.

\section{Reflection loss}

In order to investigate the absorption property, the calculated reflection loss (RL) of NC330 with different thicknesses starting from 1.6 to $2.4 \mathrm{~mm}$ has been shown in Fig. $5[11,12]$. It is obvious from the curves that RL peak shifted to lower frequency side with increasing composite thickness. This is a consequence of quarterwavelength attenuation, in which the absorption achieved the phase match criteria [12]. The optimum RL value first increases, then decreases, with increase of thickness and found $-15.3 \mathrm{~dB}$ with $1.8 \mathrm{~mm}$ of thickness at $12.2 \mathrm{GHz}$ of central frequency. Interestingly, RL reached $-13.2 \mathrm{~dB}$ with broad $2.5 \mathrm{GHz}$ of $-10 \mathrm{~dB}$ bandwidth (90\% absorption) at $9.5 \mathrm{GHz}$ of frequency using $2 \mathrm{~mm}$ of thickness. Thus, NCB with size $25 \mathrm{~nm}$ shows good absorption performance and the absorption efficiency in the measured frequency range can be tuned accordingly by varying thicknesses. Overall, the results of using $25 \mathrm{~nm}$ sized carbon black in epoxy media demonstrate that this material could be used as a microwave absorbing applications. 


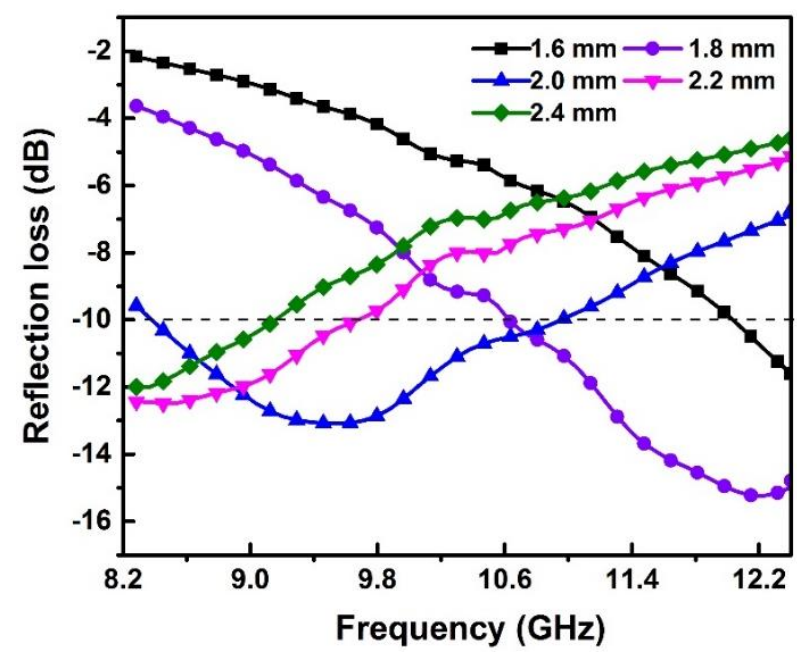

Fig. 5. Reflection loss curves with different thicknesses in the frequency ranging from 8.2-12.4 GHz for NC330.

\section{Conclusions}

In this work, the impact of different particle sizes on the EM properties of composites comprising of NCB and epoxy matrix is investigated in frequency range of 8.2-12.4 GHz. In order to identify the impurity phases in NCB powders, XRD is executed wherein no additional peaks regarding impurities were found. The agglomeration of particles with size $15 \mathrm{~nm}$ was disclosed by SEM analysis, which is attributed to high surface area of nano parties. The results of complex permittivity show that EM parameters of composites strongly depend on the particle size. The EM properties of NCB/epoxy composites increase to a certain extent and then reduce, with decrease in particle size. The particle size dependent tangent loss of NCB/epoxy composites shows that high loss characteristics is achievable with $25 \mathrm{~nm}$ size, whereas, losses decreases with size $<25 \mathrm{~nm}$ due to high surface area of the particles. In addition, NCB/epoxy composite with $25 \mathrm{~nm}$ particle size exhibited considerable EM absorption capability at a frequency of $9.5 \mathrm{GHz}$, with RL reaching $-13.2 \mathrm{~dB}$ and reflection loss below $-10 \mathrm{~dB}$ up to $2.5 \mathrm{GHz}$ with thickness of $2.0 \mathrm{~mm}$. Thus, the aforementioned findings suggest that this material can be used in aircraft and stealth technology.

\section{Acknowledgements}

The authors acknowledge the Department of Materials Science Programme, Indian Institute of Technology Kanpur.

\section{References}

1. Panwar R.; Puthucheri S.; Singh D.; and Agarwala V. IEEE Trans. Magn. 2015, 51, 2802804.

2. Wu N.; Liu X.; Zhao C.; Cui C.; and Xia A. J Alloys Compd. 2015, 656, 628 .

3. Zhou P.H.; Deng L.J.; Xie J.L.; Liang D.F.; Chen L. and Zhao X.Q. J. Magn. Magn. Mater. 2005, 292, 325.

4. Liu L.; Duann Y.; Guo J.; Chen L. and Liu S. Physica B. 2011, 406, 2261

5. Kazemzadeh A. IEEE Trans. Antennas. Propag. 2011, 59, 135

6. Rajan A. S.; Sampath S. and Shukla A. K., Energy Environ. Sci. 2014, 7, 1110 .
7. Gautam R. and Kar K. K. JMEST. 2015, 2, 2.

8. Zhao T.; Hou C.; Zhang H.; Zhu R.; She S.; Wang J.; Li T.; Liu Z. and Wei B. Sci. Rep. 2014, 4, 5619.

9. K. Singh, A. Ohlan, V. H. Pham, S. Varshney, J. Jang, S. H. Hur, W. M. Choi, M. Kumar, S. K. Dhawan, B. S. Kong and J. S. Chung, Nanoscale, 2013, 5, 2411.

10. Wang Z. and Zhao G. L., J. Compos. Mater. 2013, 3, 17.

11. Singh S. K.; Yadav A. K.; Akhtar M. J. and Kar K. K., Adv Mat Proceedings, 2017, 2, 2.

12. Jia X.; Wang J.; Zhu X.; Wang T.; Yang F.; Dong W.; Wang G.; Yang H. and Wei F., J Alloys Compd. 2017, 697, 138. 